\title{
AUTOMATIC EXTRACTION OF A SUBNETWORK FOR DYNAMIC TRAFFIC ASSIGNMENT
}

\author{
A. CHEN \& R. MACHEMEHL \\ Department of Civil, Architectural and Environmental Engineering, USA.
}

\begin{abstract}
Dynamic traffic assignment (DTA) is a commonly used approach for the process of assigning traffic from origin-destination (OD) pairs to actual roadway paths. The amount of computational effort required by the typical DTA application is approximately proportional to the square of the number of OD pairs, so for large networks the level of effort is very large. However, many network questions only involve changing one or a small number of links and therefore do not involve a very large fraction of the whole network. The concept of using a subnetwork to replace a regional network has been often used as a solution to this problem. However, how to define the size of the subnetwork, how large subnetwork is appropriate and how to build a subnetwork have been the questions. This study reviewed the researches which focused on the above questions. Then, based on the literature review, we developed a programme that can automatically build an optimal size of the subnetwork with acceptable error in Visual Interactive System for Transportation algorithms (VISTA), a mesoscopic DTA simulator. This automatic programme makes the process of examining the subnetwork size easy and is expected to have important implications for future research on DTA.

Keywords: ArcGIS, automatic, dynamic traffic assignment, subnetwork, VISTA.
\end{abstract}

\section{INTRODUCTION}

Simulation is a way to imitate how the real world progresses. This is a cost-effective way to predict and evaluate the impact of implementing changes in the real world. The simulation of traffic assignment models drivers' route choice to determine shortest travel time from origins to destinations. The requirements for simulating traffic assignment include building a simulated network similar to real-world road network and establishing an origin-destination (OD) matrix, which is the number of vehicles travelling from origins to destinations during specified departure time intervals. The representation of the simulated network (such as link volume or travel time) is comparable to the real world; therefore, the impact from any modification in the simulated network (such as setting up a work zone) would also be similar to the impact of setting up a work zone in real world.

Two rather different approaches are commonly used in the process of assigning traffic from OD pairs to actual roadway paths. These include static traffic assignment (STA) and dynamic traffic assignment (DTA). Usually, less detailed networks are simulated with STA, whereas more detailed networks are simulated with DTA. STA assumes that the inflow of a link should be equal to the outflow of a link during the same period and allows link volumes to exceed link capacity. Although the STA process can be applied to large networks efficiently, from a computational standpoint the resulting path assignments are not always realistic. The path assignments tend to characterize travel demand paths rather than link traffic volumes because in reality traffic flow cannot be higher than capacity. Also, STA

This paper is part of the proceedings of the 22nd International Conference on Urban Transport and the Environment (Urban Transport 2016)

www.witconferences.com
} 
starts to simulate congestion when the link volume is approximately equal or more than the link capacity, but in reality congestion starts at the upstream end when the upstream outflow cannot fully enter downstream. In addition, since STA assumes that inflow of the link equals the outflow of the link, the route travel time is the sum of link travel times. The route travel time does not consider the departure time, which is unrealistic because the departure time affects the route travel time. Nevertheless, experts can use a less detailed 'schematic' network to find traffic demands.

Conversely, DTA is a more appropriate choice for simulating a detailed network. DTA does not assume that inflow of a link equals to outflow of a link. In DTA, congestion occurs when inflow of a link is larger than outflow of the same link in the same period. If this is the case, then the density of the link increases while the speed decreases (Chiu et al. [1]). Consequently, DTA provides realistic results in terms of link flows that actually approximate traffic volumes. In addition, DTA predicts traffic conditions caused by the effects of (1) establishing work zones, (2) changing timing of traffic signals or (3) changing road infrastructure. The DTA process is characterized by iterative simulations of travel time changes as more traffic is assigned. The simulation time could take up to a week to run a single simulation of a large urban network. For the Dallas-Fort Worth network, the computational time is more than 2 weeks (Gemar [2]). It might not be feasible to run every single alternative that may improve traffic conditions because studying each alternative, in the case of the Dallas-Fort Worth, would take more than 2 weeks.

On the other hand, the impact from some network modifications, such as a work zone, might merely affect only a portion of the whole network. Therefore, the concept of subnetwork was created to lessen computational burden. Although subnetworks can reduce the computational time, determining the appropriate size of the subnetwork is an issue. The representation from a small network might not reflect reality because of the limited route choices. Conversely, if the OD matrix remains similar to that of the whole network, then the potential reduction in simulation time is limited.

\section{BACKGROUND}

A subnetwork represents a fraction of the entire network. The target link is shown in yellow, the subnetwork is shown in red and the entire network is shown in red and green. Gemar [2] compared the computational time and space in the full Dallas-Fort Worth network to the Dallas subnetwork and found that the time and the space were dramatically decreased.

Tables 1 and 2 (from Gemar [2]) show that the computational time and effort are reduced from approximately 1,022 $\mathrm{h}$ and $57 \mathrm{~GB}$ in the full Dallas-Fort Worth network to around $73 \mathrm{~h}$ and $10 \mathrm{~GB}$ in the Dallas subnetwork because of the reduced number of vehicles, links and nodes, even though the number of iterations in the subnetwork is more than the entire network. Also, Chen et al. [6] found that the increase of the network size results in the exponential increase of computational time. Since the subnetwork shortens the computational time and effort, we hypothesized that the subnetwork could replace the need for using the entire network. However, the accuracy of the performance of the subnetwork is an issue that requires careful consideration.

The size of the subnetwork is an issue when a property of the target link is changed (e.g. the capacity reduction). The changed target links cause rerouting of some vehicles that cannot travel through the closed-off links. As a result, the small subnetwork size might not cover all the possible rerouting options. But on the other hand, the full network size has a much 
Table 1: Example of the reduction of simulation time in Dallas-Fort Worth network (from Gemar [2]).

\begin{tabular}{|c|c|c|c|c|c|}
\hline Network & $\begin{array}{l}\text { DTA analy- } \\
\text { sis processes e }\end{array}$ & $\begin{array}{l}\text { Path gen- } \\
\text { eration itera- } \\
\text { tions }\end{array}$ & $\begin{array}{l}\text { DUE } \\
\text { terations }\end{array}$ & $\begin{array}{l}\text { Time to complete } \\
\text { analysis (hours) }\end{array}$ & $\begin{array}{l}\text { Calendar days } \\
\text { to complete }\end{array}$ \\
\hline Full DFW network & 32 & 8 & 36 & $1,022.3$ & 47 \\
\hline $\begin{array}{l}\text { Dallas subnetwork - } \\
\text { base }\end{array}$ & 15 & 20 & 40 & 72.2 & 5 \\
\hline $\begin{array}{l}\text { Dallas subnetwork - } \\
\text { impact }\end{array}$ & 12 & 20 & 40 & 73.8 & 5 \\
\hline
\end{tabular}

Table 2: Example of the reduction of file space in Dallas-Fort Worth network (from Gemar [2]).

\begin{tabular}{|c|c|c|c|c|c|}
\hline Network & $\begin{array}{c}\text { Number of } \\
\text { links }\end{array}$ & $\begin{array}{c}\text { Number of } \\
\text { nodes }\end{array}$ & $\begin{array}{c}\text { Number of } \\
\text { vehicles }\end{array}$ & $\begin{array}{l}\text { Final gap } \\
\text { measure }\end{array}$ & $\begin{array}{l}\text { Total file space } \\
\text { required }\end{array}$ \\
\hline Full DFW network & 71,721 & 31,364 & $2,512,462$ & 13.00 & $57.24 \mathrm{~GB}$ \\
\hline Dallas subnetwork - base & 16,434 & 7,408 & 858,613 & 7.15 & $9.73 \mathrm{~GB}$ \\
\hline Dallas subnetwork - impact & 16,414 & 7,394 & 858,613 & 7.80 & $10.17 \mathrm{~GB}$ \\
\hline
\end{tabular}

longer simulation time, which can make the subnetwork a more attractive option even with the missed reroutes.

Chen et al. [3] called the area of rerouting as the impacted area, whose size was defined by size parameter. The subnetwork with a size parameter of one includes a target link and all the links adjacent to the target link. The subnetwork with a size parameter of two includes the network with a size parameter of one and the links adjacent to this network (Fig. 1). The links of the next size parameter are the connected links to the links in the previous size parameter. To balance the accuracy and the simulation time, the author also specified the range of the size parameter to be from 4 to 10 . If the size parameter is less than 4 , then most critical links cannot totally be contained in the subnetwork. Additionally, if the size parameter is larger than 10, then the computational time-savings are not notably better. After the size of the subnetwork is defined, the measure that defines the performance of the subnetwork can be studied.

As suggested by Chen et al. [3], Bringardner et al. [4] investigated the measures that could evaluate the quality of the size of the subnetwork. Root mean square error (RMSE), mean censored absolute percent error and structural similarity index were examined by the comparison of the base sub OD matrix to the impacted sub OD matrix. These authors found that RMSE was the most effective measure relative to the other measures because RMSE has the ability to show a significant error difference between the impacted and base OD matrix of the subnetwork and other measures could not. Both studies used RMSE to evaluate constructing a subnetwork and to study what size of subnetwork can sufficiently replace the need to use the entire network. 


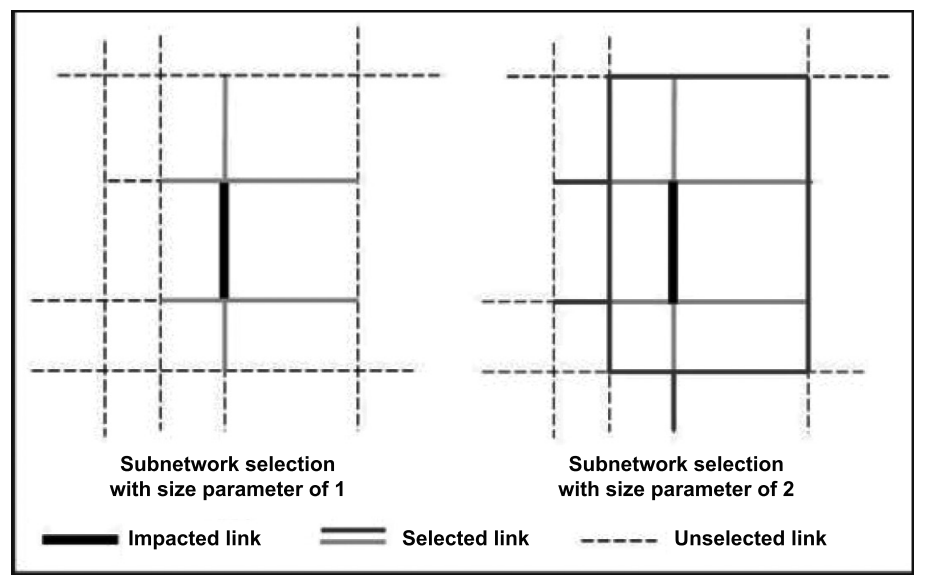

Figure 1: Visualization of the subnetwork selection with size parameter process (from Ge$\operatorname{mar}[2])$.

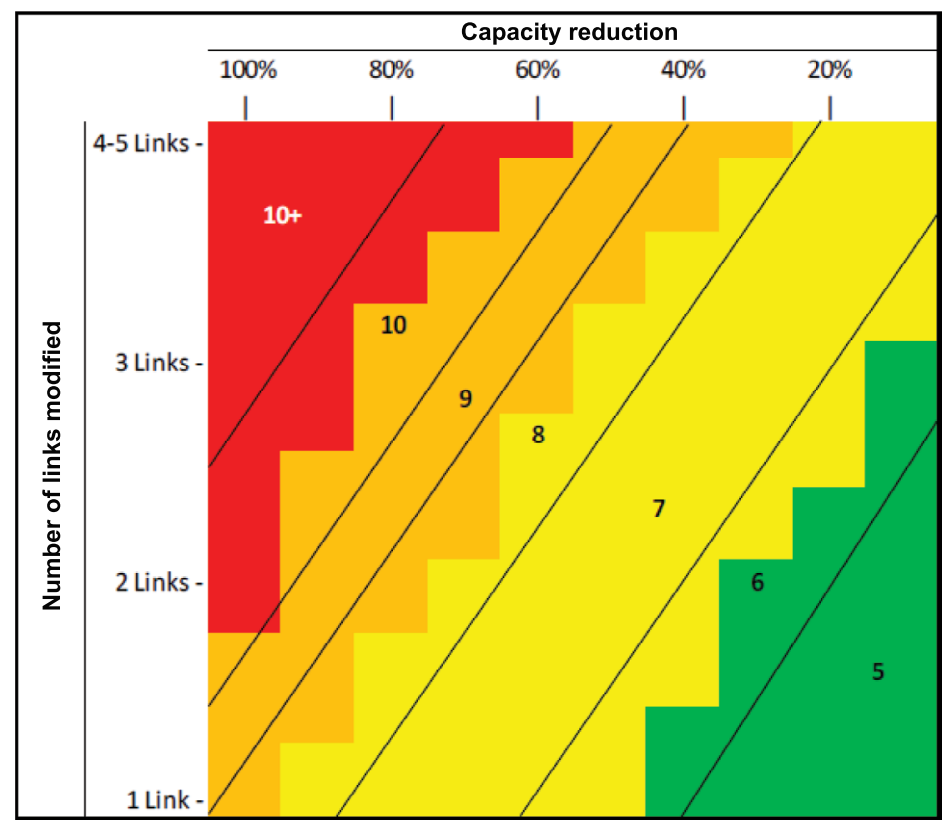

Figure 2: Recommended subnetwork sizes (from Gemar [2]).

Gemar [2] studied the relationship between the capacity reduction of the target links, the number of target links and the impacted area (Fig. 2). As the number of target links or the capacity reduction of the target links increases, the size of the subnetwork increases until the impact on the entire network is optimally represented.

In some cases, the reduction of computational time might be more important than the accuracy of the results from the subnetwork. For example, in the case of facing a deadline 
sooner than it takes to complete a full run, it may be true that a less-accurate subnetwork result is better than no result. Thus, it is important to study the balance between the accuracy from the subnetwork and the reduced time of the simulation. Bringardner [5] referred Gemar's [2] process of developing a subnetwork database and used the results to develop RMSE models, identifying the optimal subnetwork size with acceptable error. Defining the acceptable error helps quantify how much to reduce the subnetwork size and therefore increase computational efficiency. Equations (1) and (2) correspond to the base and impacted scenarios, respectively.

$$
\begin{aligned}
\text { Base }_{\mathrm{RMSE}}= & -0.115^{*} \text { size parameter }-0.018 * \text { capacity } / 1,000 \\
& -15.487 * \text { volume to capacity ratio }+6.004
\end{aligned}
$$

$$
\begin{aligned}
& \text { Impacted } \\
& \text { RMSE } \\
& \text { percent capacity reduced }+0.430 * \text { size parameter }+0.135 * \\
& \text { capacity } / 1,000-9.610 * \text { volume to capacity ratio }-0.016 * \\
& \text { size parameter*percent capacity reduced }
\end{aligned}
$$

From these equations, capacity is part of the link property, number of impacted links and percent capacity reduced are predetermined, and volume to capacity ratio comes from the results of the entire network. Increasing the size parameter results in the decrease of RMSE in both equations, and the decrease rate in eqn (2) is faster than eqn (1). When both the RMSEs are identical, the size parameter is optimal and has acceptable error.

After determining the size of the subnetwork, building the subnetwork and sub OD matrix is the next step. The process of developing a subnetwork and a sub OD matrix requires ArcGIS, which is a geographic information system (GIS) that works with maps and geographic information, $\mathrm{SSH}$, which is a network protocol that allows remote login and data transmission securely, and Visual Interactive System for Transportation Algorithms (VISTA), a mesoscopic DTA simulator (Gemar [2]).

Developing a subnetwork includes three steps: first, duplicate the entire network in VISTA database with SSH. Second, isolate the subnetwork's elements from the whole network with ArcGIS. Third, remove the redundant nodes and links that do not belong to the subnetwork in the copied database with SSH. The subnetwork's elements in the second step are the links, the nodes, the connectors and the centroids from the predetermined area in the whole network.

In addition, identifying the routing data with SSH establishes the sub OD matrix. The traffic counts in the sub OD matrix exclude the vehicles that do not cross the subnetwork. Also, the time from origin to destination in the sub OD matrix comes from the subnetwork arrival and subnetwork departure times. The code for developing a sub OD matrix was received from Network Modeling Center (NMC) at the Center for Transportation Research (CTR) at The University of Texas at Austin. This code needs to include the location of the whole network and the subnetwork before executing the SSH code. After that, the new sub OD matrix is created in the database of the subnetwork. Finally, the database of the subnetwork is completed. 


\section{PROBLEM STATEMENTS AND OBJECTIVES}

Although 'the method of developing a subnetwork by Gemar [2]' shortens the simulation run time, we still encounter the following difficulties:

1. 3D network data are required for ArcGIS.

Most traffic simulators see links and nodes as roads and intersections. Specifically, ArcGIS determines whether an intersection exists based on whether links cross. However, a problem arises if the user imports network data that contain cross roads with different elevations as a 2D dataset rather than a 3D dataset. ArcGIS will see intersections where they do not exist, thus resulting in a more erroneous subnetwork. Therefore, a full 3D network should be built in ArcGIS before extracting a subnetwork. Figure 3 illustrates an example of a subnetwork becoming larger than expected when containing elevated crossroads. The green network is parameter size 5, the green and red is size 6, and the green, red, and blue is size 7 . In the orange circle, the two middle red links are the freeways, and the crossing green link included in parameter size 5 is an urban road. The freeway does not intersect with the urban road in reality, so these freeway links should not be recognized. However, since ArcGIS could not determine whether the crossed links actually intersect or not, these freeway links are erroneously identified as part of parameter size 6 . This error carries over and results in that the links are also actually included parameter size 7 and so forth in ArcGIS.

2. The process of developing a subnetwork involves multiple manual steps that are prone to errors.

Although the process from Gemar [2] shortens the simulation time, it requires the user to install and learn the extraction of a subnetwork through ArcGIS and the transfer of a subnetwork's link and node data through SSH. In addition, developing a subnetwork with this process can easily lead to mistakes because it involves in lots of manual effort and time. This process not only involves the access of ArcGIS and SSH, but modifying JavaScript code from the MPO to build a sub OD matrix is also necessary. Therefore, this process might not be widely used and easily adapted without simplification. Also, it might not be

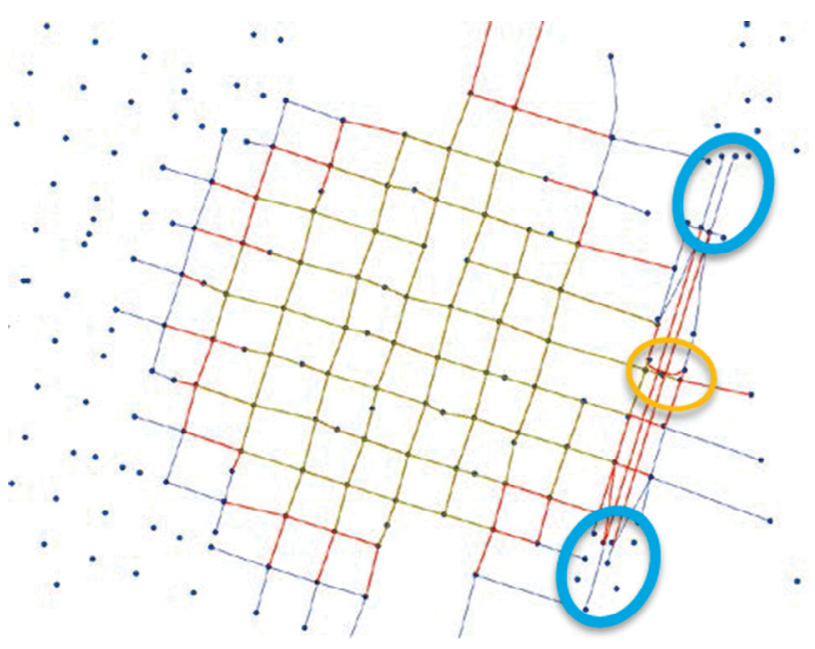

Figure 3: An elevated crossroad is misrepresented as an intersection in ArcGIS. 
possible to examine the RMSE model from Bringardner [5] that has been improved for other networks.

This study aims to develop a system to improve the previous problems by developing an automatic programme that removes the need for ArcGIS. This programme has several applications, including the calculation of a subnetwork's optimal size with the predetermined acceptable error and capacity reduction from Bringardner [5], the automatic extraction of the links and nodes without ArcGIS according to the determined subnetwork size, and the development of a subnetwork database through SSH. Therefore, this programme could reduce the manual effort and time so that the implementation of subnetworks could be broadly used. The optimal subnetwork alternative to evaluate traffic conditions allows more investigation and simulation of alternatives or scenarios without the concern of the computational burden. Furthermore, diverse testing can improve the RMSE models from Bringardner [5]. Other subnetwork analysis could also be investigated by modifying the programme. Consequently, this programme not only improves the optimal scenario but also inspires the future research for the identification of subnetwork.

\section{DATA}

The data for this programme came from NMC at the CTR. The Downtown Austin dataset is from 2005 and includes all the streets (one-way, two-way), bus routes, signal timings and OD matrix. The Downtown Austin area is west of I-35, east of North Lamar Blvd, north of Cesar Chavez and south of 38th Street. Note that the I-35 freeway is coded in this set. This programme was developed by $\mathrm{VC}++$ and the subnetwork was developed in VISTA. In addition, the detailed process of developing a subnetwork database in VISTA came from Gemar and Bringardner's previous work. The RMSE models were from Bringardner [5], meaning that the subnetwork sizes defined in this study are the same and the sub OD matrix is similar. The reason why the sub OD matrix is similar rather than identical is that VISTA provides random seeds, which affects the vehicle rerouting in every simulation.

\section{METHODOLOGY}

The programme contains two subsystems, one for extracting the subnetwork geometry and the other for creating a subnetwork database through SSH and VISTA. Before implementing the programme, the programme needs the data for the entire network, containing link IDs, link ID's upstream node ID and downstream node ID, and the link properties, including the capacity, the number of lanes and speed limit. The data are used to calculate the RMSE values and to isolate the link and node data. In addition, it is necessary to enter the impacted scenario in this programme, including the target link IDs, the capacity reduction of the target links, the acceptable RMSE and the regional network which is going to be extracted through SSH. Then, the programme runs the following procedure.

Procedure for the automatic programme

Input: The regional network and the impacted scenario First subsystem:

1. The optimal subnetwork size with acceptable error

2. The subnetwork's link and node data

Second subsystem: The subnetwork's OD matrix

Output: The subnetwork database 


\subsection{First subsystem}

In the first step, this programme refers to Bringardner's [5] models to calculate the optimal subnetwork size with an acceptable error percent. The RMSEs are affected by the size parameter only since the other parameters (percent capacity reduce, number of impacted links, etc.) are defined by the user. The size parameter starts from one until the RMSEs in the base and the impacted model are equal. Once they are equal, the optimal subnetwork size is determined.

In the second step, the programme searches for link numbers based on the determined subnetwork size. The connected links are identified by their node IDs because connected links share the same node. This method helps avoid the previously encountered problem of incorrectly identifying crossroads as intersections, as seen in ArcGIS before. After isolating the subnetwork's link and node data, the connecters and the centroids attached to the subnetwork area are also isolated. Finally, the link data (including the links and connectors) and the node data (including the nodes and centroids) for the subnetwork are complete.

\subsection{Second subsystem}

In this subsystem, SSH copies the regional network database to the subnetwork database, including the full network OD matrix and the vehicle tracking to the subnetwork. Then, this programme replaces the entire network's link and node data with the subnetwork's data. The full network OD matrix is also replaced using the modified JavaScript code for extracting a sub OD matrix from the full OD matrix. The modification includes the location of the regional OD matrix and the subnetwork's link data. After building the subnetwork geometry and the sub OD matrix in VISTA, the development of the subnetwork database is finished. Figure 4 shows the output after using the automatic programme; the target links are three and have $50 \%$ capacity reduction at Guadalupe Street (orange line).

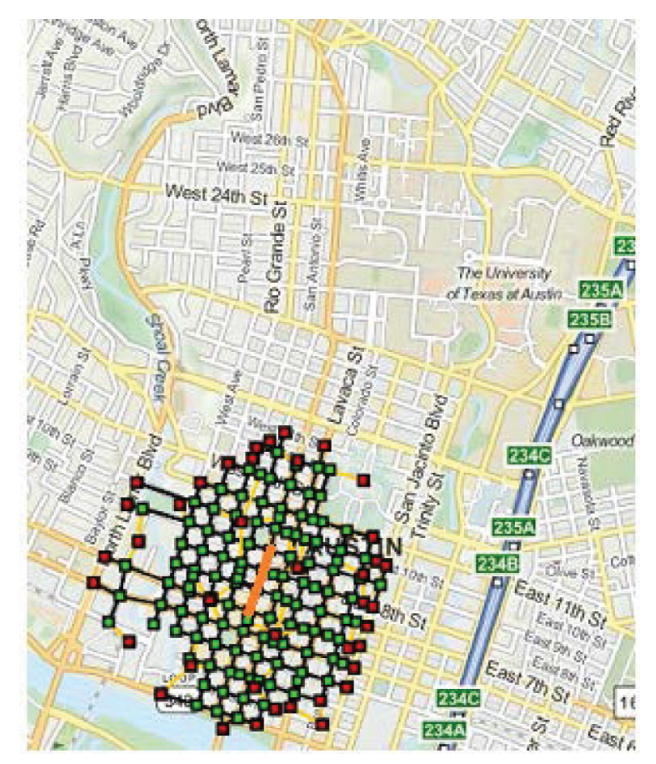

Figure 4: Example of a subnetwork developed with the automatic programme. 
Table 3: The number of links based on the parameter size and the work zone size according to the scenario.

\begin{tabular}{lrrrrcrcrcc}
\hline Bringardner [5] & \multicolumn{3}{c}{} & \multicolumn{3}{c}{ Auto programme } & \multicolumn{3}{c}{ Difference } \\
\hline 15 th & \multicolumn{3}{c}{ Order } & \multicolumn{3}{c}{ Order } & \multicolumn{3}{c}{ Order } \\
\hline Work zone & 7 & 9 & 11 & 7 & 9 & 11 & 7 & 9 & 11 \\
1 & 396 & 595 & - & 388 & 588 & - & 8 & 7 & - \\
2 & 425 & 645 & 848 & 420 & 638 & 825 & 5 & 7 & 23 \\
3 & 442 & 660 & 876 & 437 & 649 & 847 & 5 & 11 & 29 \\
7 th & & Order & & & Order & & & Order & \\
Work zone & 7 & 9 & 11 & 7 & 9 & 11 & 7 & 9 & 11 \\
1 & 371 & 572 & - & 362 & 559 & - & 9 & 13 & - \\
2 & 404 & 584 & 714 & - & 568 & 694 & - & 16 & 20 \\
3 & 420 & 598 & - & 407 & 582 & - & 13 & 16 & - \\
Guadalupe & & Order & & & Order & & & Order & \\
Work zone & 7 & 9 & 11 & 7 & 9 & 11 & 7 & 9 & 11 \\
1 & 368 & 539 & - & 368 & 539 & - & 0 & 0 & - \\
2 & 402 & 569 & 807 & 402 & 569 & 807 & 0 & 0 & 0 \\
3 & 448 & 625 & 824 & 448 & 625 & 824 & 0 & 0 & 0 \\
\hline
\end{tabular}

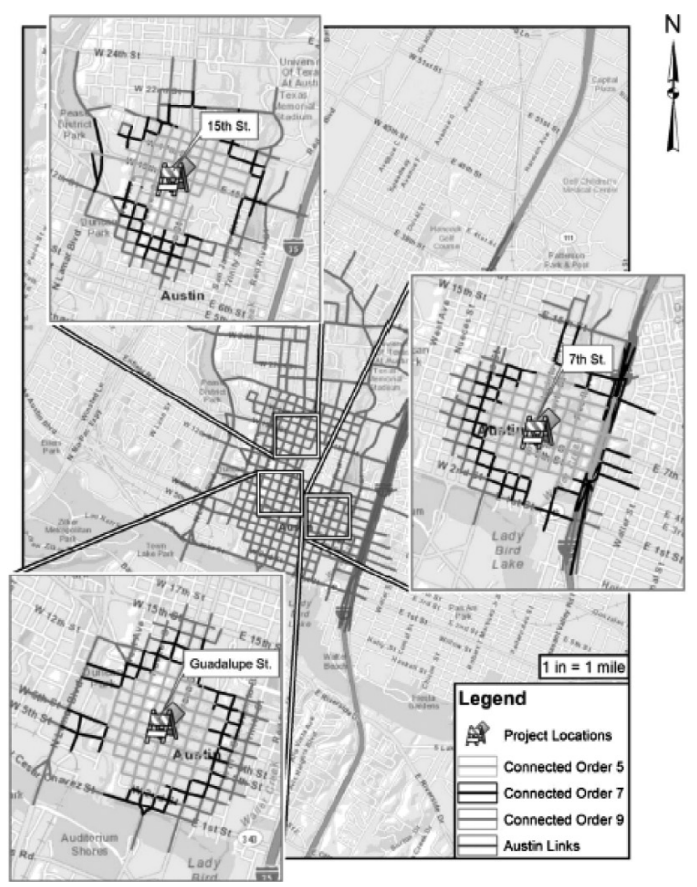

Figure 5: Locations chosen for the case study. 


\section{CASE STUDY}

This study uses the Downtown Austin network as the case study. The target links (Guadalupe, 7th, and 15th) and the parameter sizes (7,9 and 11) are identical to Bringardner's [5] work to validate that the automatic programme followed his procedure.

The link data are shown in Table 3. The numbers of links are identical in the Guadalupe Street scenario but are different in 7th Street and 15th Street scenarios. The subnetwork only includes urban streets in the Guadalupe Street scenario. The 7th Street and 15th Street scenarios have covered the freeway links with parameter size 7. Since ArcGIS cannot see the elevation of the crossroads, the number of links in 7 parameter size in ArcGIS is more than that in the automatic programme. The difference between the number of links in Bringardner's work and the new automatic programme increases in 7th and 15th Street scenarios when the work zone size and the parameter size increase (Fig. 5).

\section{CONCLUSIONS AND FUTURE RESEARCH}

This article implements an operation that automatically develops a subnetwork database in VISTA. The programme inputs are the data of the full network and the impacted scenario, including the target link, its capacity reduction and the acceptable error. The programme output is the subnetwork database. This programme has the ability to simplify the manual process and to reduce the possibility of manual mistakes. Additionally, the reduction in simulation time allows for opportunity to discuss and analyse more alternatives to improve the traffic condition in a shorter time. Furthermore, new avenues of research on subnetwork analysis could be opened up due to the convenience of using this programme.

This study referred to Bringardner's [5] RMSE models to define the most effective rerouting area as the subnetwork. The models were examined in one-way urban streets. However, other types of roads, such as arterials, might affect the rerouting area. In this case, the arterial capacity is larger than the nearby parallel streets, so the impact of reducing capacity on the subnetwork size might be larger than before because the nearby streets might not be able to afford accommodating the number of rerouting vehicles. The models should be examined with different types of road or be considered with other parameters to make this model more applicable to general use in the future, such as the capacity ratio of the target links and the nearby links or the speed limit of the target link.

Also, the data used to develop the RMSE model were PM peak-hour data in Downtown Austin, so the total demand was relatively large. The heavy demand results in the larger rerouting area because the other parallel streets were also congested. On the other hand, the impacted area would be smaller at off-peak hours and would lessen the computational burden. The total demand could also be taken into consideration to improve the models.

In addition, the RMSE models are investigated in the Downtown Austin network and the Dallas network, which include more one-way streets. These networks might be simpler than other networks that include two-way street, so the size of impacted area might be more or less than other types of networks. Therefore, these models should be examined in more diverse networks in the future.

\section{REFERENCES}

[1] Chiu, Y.C., Bottom, J., Mahut, M., Paz, A., Balakrishna, R., Waller, T. \& Hicks, J., A primer for dynamic traffic assignment. Transportation Research Board, 2-3, pp. 244-245, 2010.

[2] Gemar, M.D., Subnetwork Analysis for Dynamic Traffic Assignment: Methodology and Application. PhD dissertation, University of Texas at Austin, 2013. 
[3] Chen, B.Y., Lam, W.H., Sumalee, A., Li, Q. \& Li, Z.C., Vulnerability analysis for largescale and congested road networks with demand uncertainty. Transportation Research Part A: Policy and Practice, 46(3), pp. 501-516, 2012. DOI: 10.1016/j.tra.2011.11.018.

[4] Bringardner, J.W., Gemar, M.D., Boyles, S.D. \& Machemehl, R.B. Establishing the Variation of Dynamic Traffic Assignment Results Using Subnetwork Origin-Destination Matrices. In Transportation Research Board 93rd Annual Meeting, no. 14-3107, 2014.

[5] Bringardner, J.W., Application of a subnetwork characterization methodology for dynamic traffic assignment, Dissertation, 2014.

[6] Chen, A., Lee, D.H. \& Jayakrishnan, R., Computational study of state-of-the-art pathbased traffic assignment algorithms. Mathematics and Computers in Simulation, 59(6), pp. 509-518, 2002. DOI: 10.1016/S0378-4754(01)00437-2. 